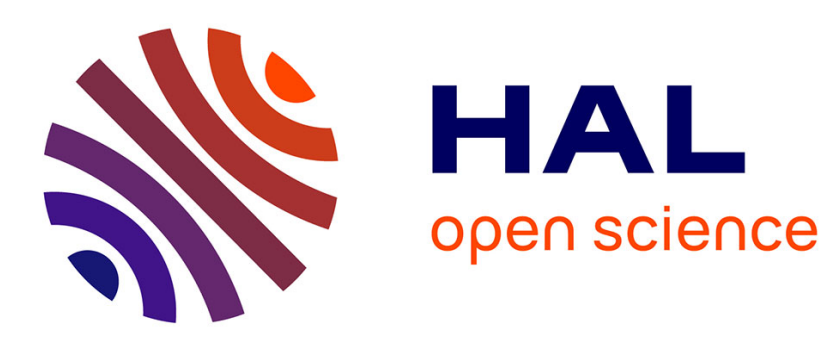

\title{
Optimal secondary energy growth in a plane channel flow
}

\author{
Carlo Cossu, M. Chevalier, D.S. Henningson
}

\section{To cite this version:}

Carlo Cossu, M. Chevalier, D.S. Henningson. Optimal secondary energy growth in a plane channel flow. Physics of Fluids, 2007, 19 (5), pp.058107. 10.1063/1.2736678 . hal-01023335

\section{HAL Id: hal-01023335 \\ https: / hal-polytechnique.archives-ouvertes.fr/hal-01023335}

Submitted on $20 \mathrm{Jul} 2014$

HAL is a multi-disciplinary open access archive for the deposit and dissemination of scientific research documents, whether they are published or not. The documents may come from teaching and research institutions in France or abroad, or from public or private research centers.
L'archive ouverte pluridisciplinaire HAL, est destinée au dépôt et à la diffusion de documents scientifiques de niveau recherche, publiés ou non, émanant des établissements d'enseignement et de recherche français ou étrangers, des laboratoires publics ou privés. 


\section{AIP | Physics of Fluids}

\section{Optimal secondary energy growth in a plane channel flow}

Carlo Cossu, Mattias Chevalier, and Dan S. Henningson

Citation: Physics of Fluids (1994-present) 19, 058107 (2007); doi: 10.1063/1.2736678

View online: http://dx.doi.org/10.1063/1.2736678

View Table of Contents: http://scitation.aip.org/content/aip/journal/pof2/19/5?ver=pdfcov

Published by the AIP Publishing

\section{Articles you may be interested in}

Secondary energy growth and turbulence suppression in conducting channel flow with streamwise magnetic field Phys. Fluids 24, 074101 (2012); 10.1063/1.4731293

Three dimensional flow around a circular cylinder confined in a plane channel Phys. Fluids 23, 064106 (2011); 10.1063/1.3599703

Analytical and numerical investigations of laminar and turbulent Poiseuille-Ekman flow at different rotation rates Phys. Fluids 22, 105104 (2010); 10.1063/1.3488039

Transient growth and minimal defects: Two possible initial paths of transition to turbulence in plane shear flows Phys. Fluids 16, 3515 (2004); 10.1063/1.1775194

Linear stability of laminar plane Poiseuille flow of helium II under a nonuniform mutual friction forcing Phys. Fluids 13, 983 (2001); 10.1063/1.1352628

\section{A|P| Journal of}




\title{
Optimal secondary energy growth in a plane channel flow
}

\author{
Carlo Cossu \\ LadHyX, CNRS-École Polytechnique, F-91128 Palaiseau, France \\ Mattias Chevalier \\ The Swedish Defence Research Agency (FOI), SE-164 90 Stockholm, Sweden \\ Dan S. Henningson \\ KTH Mechanics, SE-100 44 Stockholm, Sweden
}

(Received 24 January 2007; accepted 3 April 2007; published online 30 May 2007)

\begin{abstract}
The optimal growth of perturbations to transiently growing streaks is studied in Poiseuille flow. Basic flows are generated by direct numerical simulation giving "primary" optimal spanwise periodic vortices of finite amplitude as the initial condition. They evolve into finite amplitude primary transiently growing streaks. Linear "secondary" optimal energy growth supported by these primary flows are computed using an adjoint technique which takes into full account the unsteadiness of the basic flows. Qualitative differences between primary and secondary optimal growths are found only when the primary streaks are locally unstable. For locally stable primary streaks, the secondary optimal growth has the same scalings with Reynolds number $R$ as the primary optimal growth and the maximum growth is attained by streamwise uniform vortices, suggesting that the primary and secondary optimal growth are based on the same physical mechanisms. When the primary streaks are locally unstable the secondary optimal growth of unstable wavenumbers scale differently with $R$ and the maximum growth is attained for streamwise nonuniform sinuous perturbations, indicating the prevalence of the inflectional instability mechanism. (C) 2007 American Institute of Physics. [DOI: 10.1063/1.2736678]
\end{abstract}

The flow in a plane channel is observed to be turbulent when the Reynolds number $R$ roughly exceeds 1000, a value which is well below the critical Reynolds number at which the Poiseuille solution becomes linearly unstable. The Navier-Stokes operator linearized about the Poiseuille laminar solution is highly non-normal and can sustain large transient growth of the perturbation energy even when the basic flow is linearly stable. Upon optimization it is found that the initial perturbations leading to the largest transient growth consist of spanwise periodic pairs of counter-rotating vortices uniform in the streamwise direction ${ }^{1,2}$ that induce spanwise periodic and streamwise uniform regions of excess and deficit of streamwise velocity, called streamwise streaks. The amplification of energy during this process is algebraic and can be very large, being of $O\left(R^{2}\right)$. In the presence of streaks the streamwise velocity profiles are inflectional and are subject to strong inflectional instabilities when their amplitude exceeds a critical threshold. ${ }^{3,4}$ This sequence of an algebraic primary growth followed by an exponential secondary instability is the core of the so-called "streamwise vortices" (SV) transition scenario. This subcritical transition to turbulence is triggered by initial conditions with energy much lower than the ones required in the scenarios based on subcritical finite amplitude TS waves. ${ }^{3}$ Claiming that the secondary instability of the streaks is not always required for the streaks to break down, Schoppa and Hussain ${ }^{5}$ proposed a transition scenario based on secondary transient (see also Ref. 6) algebraic growth (STG) of $x$-dependent perturbations on top of the primary streaks. This scenario, that allows transition even for low amplitude streaks, stable to modal secondary (exponen- tial) instabilities, has been validated on streaks extracted from turbulent channel flow simulations. Secondary optimal growth of energy riding on primary streaks profiles has been computed in the Blasius boundary layer, ${ }^{7}$ where it is found that very large transient growth can be supported by these streaks. In both these studies, the initial condition leading to transient growth is given on streaks of already large amplitude frozen or allowed to slowly decay by viscous diffusion. However, in the transition process the streak amplitude grows from roughly zero at $t=0$ to its maximum value, reached at a large time $t_{\max }$, when generated from streamwise vortices given as initial conditions. One may argue that, in the standard initial value problem, also the secondary initial perturbations should be given at $t=0$ and not at $t_{\max }$. The goal of the present study is to determine if large linear transient growth can survive on growing-decaying streaks. For this purpose we compute the secondary optimal linear growth supported by optimal growing-decaying streaky basic flows. ${ }^{8}$

We consider the flow at constant volume flux $Q$ per unit spanwise length of an incompressible viscous fluid of density $\rho$ and kinematic viscosity $\nu$ in a channel delimited by two plane parallel walls respectively situated at distance $\pm h$ from the channel center plane. This motion of the fluid is governed by the Navier-Stokes equations that, in dimensionless form depend on the Reynolds number $R=3 Q / 4 \nu$. No-slip boundary conditions are enforced at the walls and the initial condition $\mathbf{u}(x, y, z, 0)=\mathbf{u}_{0}(x, y, z)$ are enforced at $t=0$, where we denote by $\mathbf{u}$ the velocity made dimensionless with the dimensionless velocity $U=3 Q / 4 h$ and by $x, y$, and $z$, respectively 

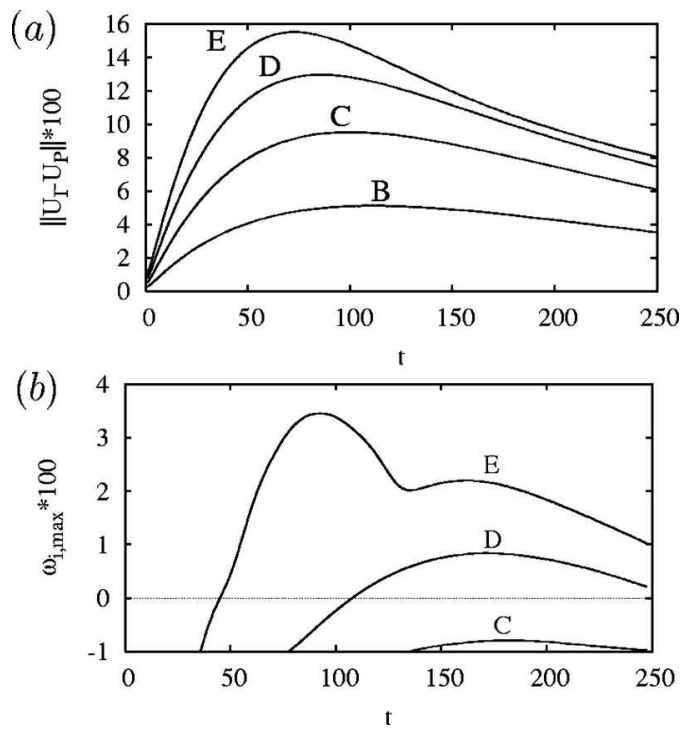

FIG. 1. (a) Temporal evolution of the amplitude $\left\|U_{I}-U_{P}\right\|$ of the departure of the primary basic flows from the Poiseuille flow. (b) Corresponding local maximum modal temporal growth rate $\omega_{i, \max }$ of the fundamental sinuous perturbations.

the streamwise, wall normal, and spanwise coordinates made dimensionless with $h$.

If a generally unsteady solution of the Navier-Stokes equations $\mathbf{U}(x, y, z, t)$ is taken as the basic flow, the generic solution $\mathbf{u}$ is decomposed into the sum of the basic flow and its perturbation $\mathbf{u}=\mathbf{U}+\mathbf{u}_{p}$. The optimal (temporal) growth $G(t, R)$ of the perturbations between the times $t=0$ and $t$ is defined as the ratio of the norm of the perturbation field at $t$ to the norm of the initial perturbation $\mathbf{u}_{p_{0}}$, optimized over all allowed initial perturbations, $G(t, R)=\sup _{\mathbf{u}_{p_{0}}}\left\|\mathbf{u}_{p}\right\| /\left\|\mathbf{u}_{p_{0}}\right\|$. The standard energy norm of the perturbations used is $\left\|\mathbf{u}_{p}\right\|$ $=\left[(1 / \mathcal{V}) \int_{\mathcal{V}} \mathbf{u}_{p} \cdot \mathbf{u}_{p} d \mathcal{V}\right]^{1 / 2}$ and the perturbations are computed from the Navier-Stokes equations linearized in the neighborhood of $\mathbf{U}$. The maximum optimal growth is defined as $G_{\text {max }}(R)=\sup _{t} G(t, R)$. We denote by $\mathbf{u}_{p}^{\text {opt })}$ the optimal initial perturbation which realizes the maximum optimal growth $G_{\max }$. We will refer to primary optimals when the Poiseuille solution is taken as basic flow and to secondary optimals when a streaky finite amplitude unsteady flow is assumed as basic flow. In the linear framework the perturbation at time $t$ is related to the initial perturbation by a linear operator $\mathbf{P}_{[t, 0]}$, called the propagator of the system, $\mathbf{u}_{p}=\mathbf{P}_{[t, 0]} \mathbf{u}_{p_{0}}$. As the norm of the perturbation is defined in terms of the inner product $\left\|\mathbf{u}_{p}\right\|=\left\langle\mathbf{u}_{p}, \mathbf{u}_{p}\right\rangle^{1 / 2}$, then $G(t, R)=\left\|\mathbf{P}_{[0, t]}^{\dagger} \mathbf{P}_{[t, 0]}\right\| \|^{1 / 2}$, where the operator $\mathbf{P}_{[0, t]}^{\dagger}$ is the adjoint of the propagator and corresponds to the backward in time integration of the adjoint linearized Navier-Stokes equations from $t$ to $t=0$. As $\mathbf{P}_{[0, t]}^{\dagger} \mathbf{P}_{[t, 0]}$ is self-adjoint, all its eigenvalues are real and its norm is its largest eigenvalue and can therefore be computed using the standard power iterations method. ${ }^{10}$ Each iteration is composed of a direct integration of the linearized equations followed by a backward in time integration of the adjoint equations. The unsteady nature of the primary basic flow is taken into full account by this procedure.

The Navier-Stokes equations are numerically solved

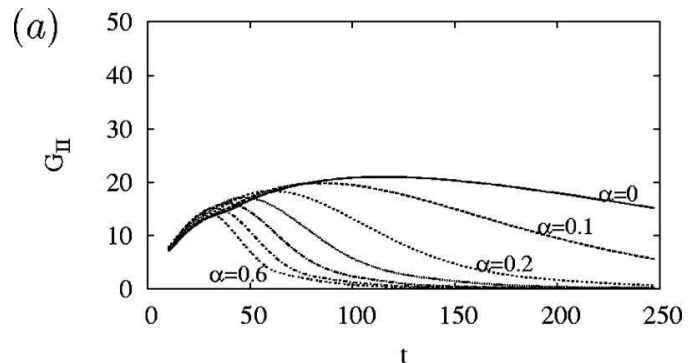

(b)

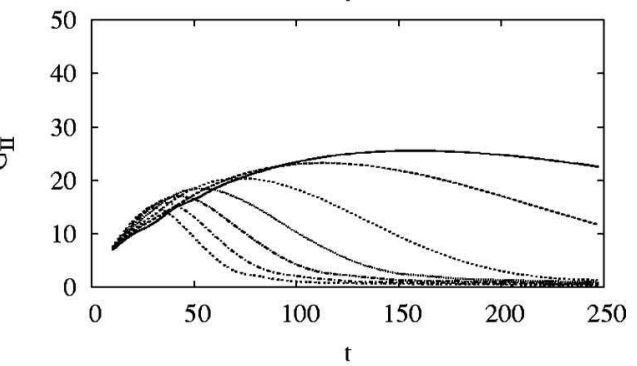

(c)
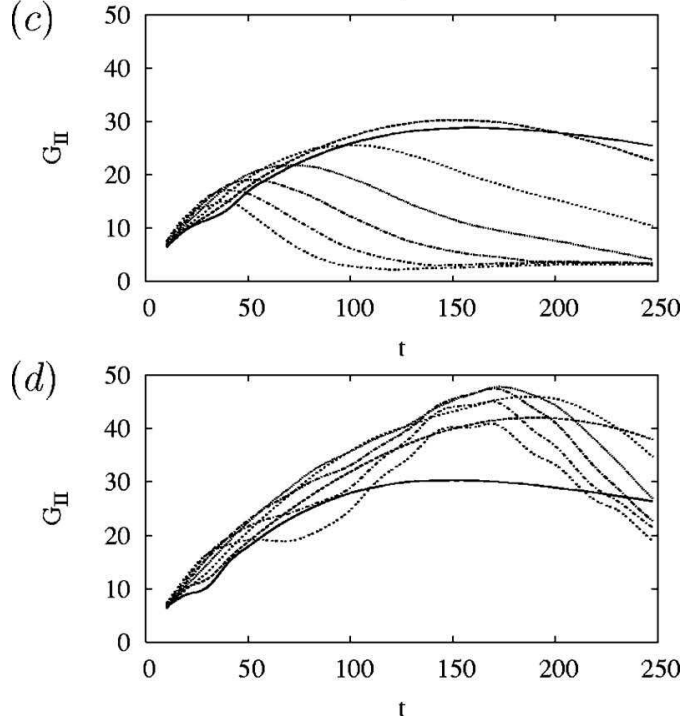

FIG. 2. Secondary optimal growths $\hat{G}_{\mathrm{II}}(t)$ with constrained streamwise wavenumbers $\alpha=0$ (solid line) and $\alpha=0.1,0.2, \ldots, 0.6$ for the basic flows (a) A, (b) C, (c) D, (d) E $(\beta=2, R=1500)$.

both to compute the basic flows and to compute the optimal perturbations with the code described e.g., in Ref. 11, to which we refer the reader for further details. The threedimensional, time dependent, incompressible Navier-Stokes equations are solved using a Fourier representation in the streamwise and spanwise directions and Chebyshev polynomials in the wall-normal direction, together with a pseudospectral treatment of the nonlinear terms. The time integration is based on a four-step low-storage third-order Runge-Kutta method for the nonlinear terms and a secondorder Crank-Nicolson method for the linear terms. This code has been recast in perturbation form to integrate the linearized equations and it includes the possibility of integrating backward in time the adjoint equations. ${ }^{12}$ The direct and the backward adjoint integrations are used in the power method to compute the optimal growth. Less than $10^{-4}$ relative error is required on $G(t)$, which is typically attained in 10-15 power iterations for the considered cases.

We first consider the already well studied Poiseuille so- 

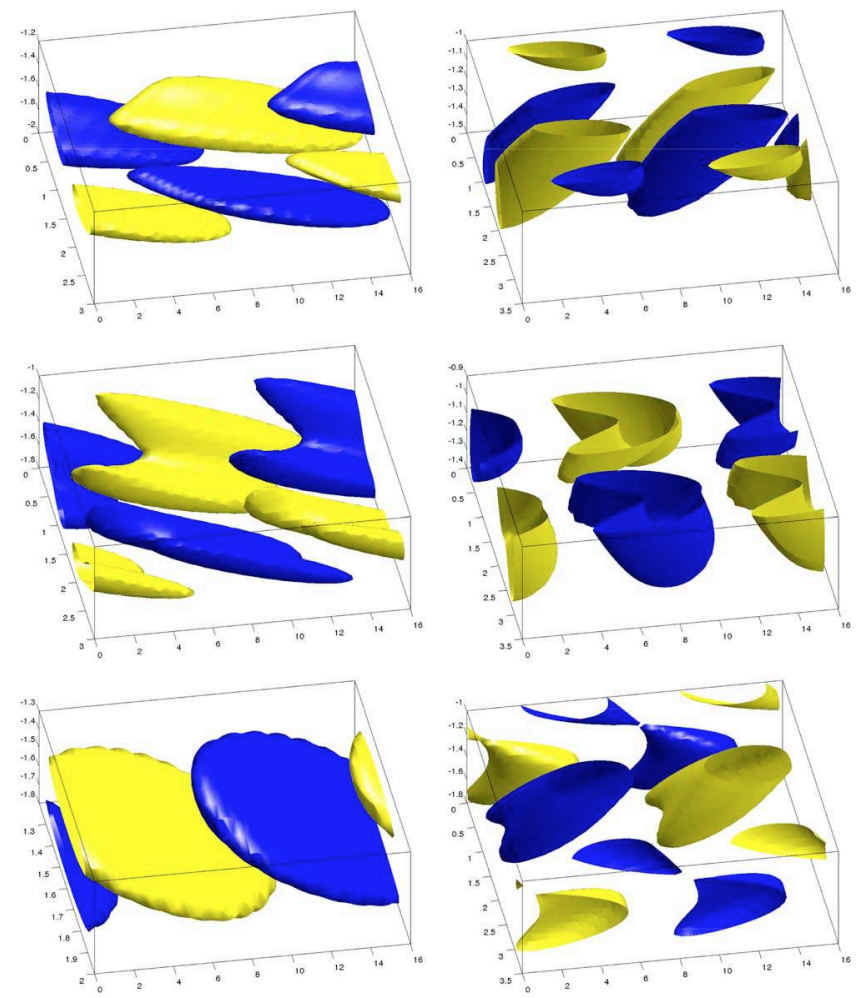

FIG. 3. (Color online) Streamwise (top), wall-normal (middle), and spanwise (bottom) velocity optimal perturbations of the E primary basic flow at $t=0$ (left column) and at the time of maximum amplification $t_{\mathrm{II}_{\max }}$ (right column). The isosurfaces of $\pm 20 \%$ of the maximum of the three velocity components are visualized in dark and light gray (blue and yellow online).

lution $\mathbf{U}_{P}(y)=\left\{1-y^{2}, 0,0\right\}$. The optimal perturbations $\mathbf{u}_{I}^{\text {(opt) }}$ of this basic flow are streamwise uniform, spanwise periodic vortices inducing a maximum growth $G_{I \max }$, at the time $t_{\text {Imax }}$, of streamwise uniform, spanwise periodic streaks. For sufficiently large Reynolds numbers the optimal streamwise $(\alpha)$ and spanwise $(\beta)$ wavenumbers are roughly $(\alpha=0, \beta=2)$ and both $G_{I \max }$ and $t_{I \max }$ scale proportionally to the Reynolds number. ${ }^{1,2,13}$

Following the same rationale used in previous studies on the stability of streaks, ${ }^{3,7,14-16}$ finite amplitude streaky basic flows are computed using as initial condition the Poiseuille flow plus the primary optimal perturbations with finite amplitude $\mathbf{U}_{I 0}(y, z)=\mathbf{U}_{P}(y)+A_{I} \mathbf{u}_{I}^{\text {(opt) }}(y, z)$, with $\left\|\mathbf{u}_{I}^{(\text {opt })}\right\|=1$. We have considered the case $\beta=2$ at $R=1500$ and the amplitudes $A_{I}=0$ (case $\mathrm{A}$, corresponding to Poiseuille flow), $A_{I}$ $=0.0025$ (case B),.., $A_{I}=0.01$ (case E). The nonlinear Navier-Stokes equations are numerically integrated providing a family of basic flows $\mathbf{U}_{I}\left(y, z, t, R, A_{I}\right)$ parametrized by $A_{I}$. These basic flows represent temporally growing and decaying nonlinear streaks uniform in the streamwise direction; their amplitudes are reported in Fig. 1(a). The modal stability properties of these basic flows are then investigated using an already validated code. ${ }^{16}$ The maximum temporal growth rate $\omega_{i, \max }$ is computed under the hypothesis that the basic flow is steady. For sufficiently large amplitudes of the streaks (cases D and E) the appearance of a local sinuous fundamental instability of the streaks is observed [see Fig. 1(b)], in accordance with previous work, ${ }^{3}$ with most amplified wave- numbers near $\alpha=1.6$. Secondary perturbations $\mathbf{u}_{\mathrm{II}}$ are defined as the deviation from the primary basic flow, $\mathbf{u}=\mathbf{U}_{\mathrm{I}}+\mathbf{u}_{\mathrm{II}}$. The optimal, $G_{\mathrm{II}}\left(t, R, A_{\mathrm{I}}\right)$, and maximum, $G_{\mathrm{IImax}}\left(R, A_{\mathrm{I}}\right)$, growth of secondary perturbations depend on the parameter $A_{\text {I }}$ identifying the basic flow under consideration. Only fundamental perturbations, having the same spanwise basic periodicity of the basic flow, are considered. As the primary basic flows are homogeneous in the streamwise direction, it is possible to compute the optimal growths $\hat{G}_{\mathrm{II}}\left(R, A_{\mathrm{I}}, \alpha\right)$ restricted to perturbations of a given streamwise wavenumber $\alpha . G_{\mathrm{II}}\left(t, R, A_{\mathrm{I}}\right)$ is the envelope of these restricted optimal curves. As mentioned, the power iterations method is used to compute $\hat{G}_{\mathrm{II}}\left(R, A_{\mathrm{I}}, \alpha\right)$. For the direct and adjoint temporal integrations performed at each iteration, a box of length $L_{x}=2 \pi / \alpha$ has been used. The number of points $N_{x}$ used in the streamwise direction has been chosen to always keep $\Delta x=L_{x} / N_{x}<1$ which is sufficient for ensuring converged results. We have therefore used $N_{x}=16$ for $\alpha=0.4,0.5$, and $0.6, N_{x}=32$ for $\alpha=0.2$ and 0.3 and $N_{x}=64$ for $\alpha=0.1$. The case $\alpha=0$ has been computed taking into account only the streamwise uniform part of the solution with $N_{x}=4$. In all the simulations $L_{z}=\pi$, corresponding to $\beta=2$ and $N_{y}=64$ and $N_{z}=16$ points have been used in, respectively, the wall normal and spanwise directions. Doubling the number of points $N_{z}$ in the spanwise direction does not affect the results in any noticeable way. The $\hat{G}_{\mathrm{II}}\left(R, A_{\mathrm{I}}, \alpha\right)$ curves are reported in Fig. 2. For cases $\mathrm{B}$ (not shown) and $\mathrm{C}$ the secondary optimal growth curves are similar to the primary optimal growth (case A) but with slightly larger magnitude; the perturbations realizing the maximum optimal growth $G_{\text {IImax }}$ are streamwise uniform $\left(\alpha_{\text {IImax }}=0\right)$. For the locally unstable case $\mathrm{D}$, however, the most amplified wavenumber is not zero $\left(\alpha_{\text {IImax }}=0.1\right)$ meaning that the most amplified secondary perturbations are now the sinuous ones. For case $\mathrm{E}$ all the considered wavenumbers $\alpha=0.1$ to 0.6 are more amplified than $\alpha=0$. These most amplified wavenumbers optimally conciliate the non-normal initial growth, larger for low wavenumbers, with the late growth due to local instability, which is larger for larger wavenumbers.

The three velocity components of the optimal secondary perturbations of the primary basic flow E are reported in Fig. 3 in the bottom half of the channel (the top half is symmetric with respect to the center-channel plane). Their streamwise wavenumber is $\alpha_{\text {IImax }}=0.4$ [see Fig. 2(c)]. From the spanwise symmetry of the spanwise velocity components and the antisymmetry of the streamwise and wall-normal velocity components the sinuous nature of the secondary optimal is easily recognized. At $t=0$ the three velocity components of the secondary optimal perturbations are tilted upstream. At the time of maximum secondary amplification $t_{\text {IImax }}$, the secondary optimal perturbations are tilted in the downstream direction, with the shear, except the wall normal velocity component that is oriented roughly normal to the wall. The same upstream/downstream tilting has been observed in the case of the Blasius boundary layer ${ }^{7}$ and attributed to a modified Orr mechanism. Further calculations showed that with increasing amplitude $A_{\mathrm{I}}$ of the primary vortices (and of the induced streaks), the secondary optimal perturbations de- 

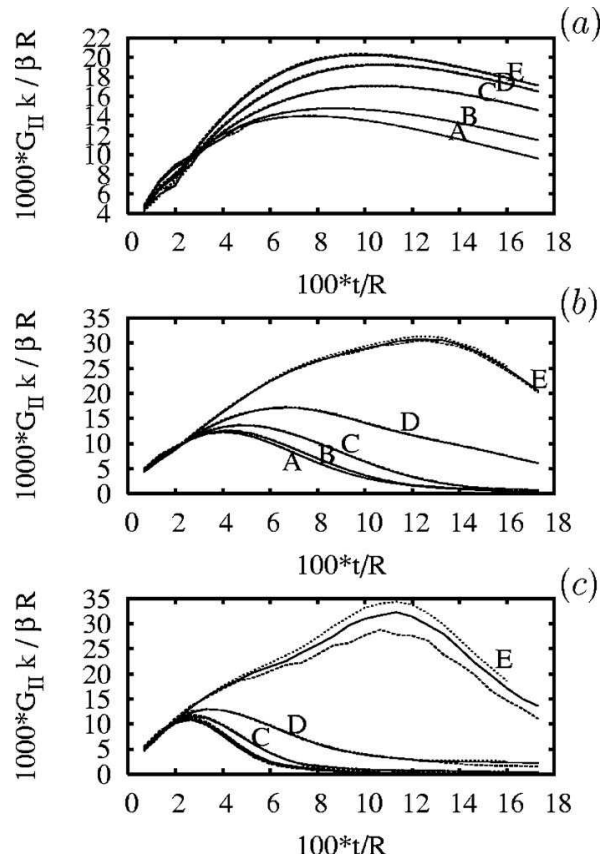

FIG. 4. Rescaled secondary optimal growths restricted to the selected rescaled streamwise wavenumbers $\alpha R=0$ (a), $\alpha R=300$ (b), and $\alpha R=600$ (b) for the primary basic flows A, B, C, D, and E (bottom to top). Data computed at $R=750, R=1500$, and $R=3000$ are reported as, respectively, dashed, solid, and dotted lines.

crease their streamwise wavelength and tend to concentrate near the regions of maximum spanwise shear of the streaks.

Since the secondary optimal growths look very similar to the primary growths, we investigate if they obey the same scalings with $R$ that apply to the primary optimals. The secondary optimal growths have been recomputed at two additional Reynolds numbers $R=750$ and $R=3000$ on the rescaled basic flows having the same $A_{\mathrm{I}} R$. In Fig. 4 the rescaled optimal secondary growth $k \hat{G}_{\text {II }} /(\beta R)$ is plotted versus the rescaled time $t / R$ for corresponding rescaled wavenumbers $\alpha R$ and $k=\left(\alpha^{2}+\beta^{2}\right)^{1 / 2}$. The rescaled growths coincide, except for the locally unstable D and E basic flows where the growth increases more with $R$ for the locally unstable streamwise wavenumbers. In the absence of local sinuous inflectional instability, this indicates that secondary growth is likely to be due to the same physical mechanisms as the primary ones, i.e., the so-called lift-up effect.

We have found that the optimal growth of secondary perturbations riding on the streaks can survive the growth phase of the primary streaks. As long as the primary streaks are locally stable, the secondary optimal growth is very similar to primary growth observed in the absence of streaks, it also scales with $R$ just as the primary optimal growth and the optimal perturbations are streamwise uniform. The fact that streamwise nonuniform perturbations can be strongly amplified in this regime is not surprising because the same type of amplification already exists in the absence of streaks [compare, for instance, Figs. 2(a) and 2(b)]. For streaks of larger amplitude that are locally unstable to streamwise dependent perturbations, the optimal perturbations realizing the maximum growth $G_{\mathrm{II}_{\max }}$ are streamwise dependent and the optimal growth do not scale any more with $R$ as the primary growth leading to much larger growths.

${ }^{1}$ K. M. Butler and B. F. Farrell, "Three-dimensional optimal perturbations in viscous shear flow," Phys. Fluids A 4, 1637 (1992).

${ }^{2}$ S. C. Reddy and D. S. Henningson, "Energy growth in viscous channel flows," J. Fluid Mech. 252, 209 (1993).

${ }^{3}$ S. C. Reddy, P. J. Schmid, J. S. Baggett, and D. S. Henningson, "On the stability of streamwise streaks and transition thresholds in plane channel flows," J. Fluid Mech. 365, 269 (1998)

${ }^{4}$ F. Waleffe, "Hydrodynamic stability and turbulence: Beyond transients to a self-sustaining process," Stud. Appl. Math. 95, 319 (1995).

${ }^{5}$ W. Schoppa and F. Hussain, "Coherent structure generation in near-wall turbulence," J. Fluid Mech. 453, 57 (2002).

${ }^{6}$ S. Grosssmann, "The onset of shear flow turbulence," Rev. Mod. Phys. 72, 603 (2000).

${ }^{7}$ J. Hœpffner, L. Brandt, and D. S. Henningson, "Transient growth on boundary layer streaks," J. Fluid Mech. 537, 91 (2005).

${ }^{8}$ This kind of analysis is standard in nonlinear dynamical systems theory where the logarithm of the secondary optimal growth divided by $t$ is known as the finite time Lyapunov exponent (see Ref. 9).

${ }^{9}$ P. Manneville, Dissipative Structures and Weak Turbulence, Perspective in Physics (Academic, New York, 1990).

${ }^{10}$ If the (real) largest eigenvalue $\lambda^{*}$ of $\mathbf{H}=\mathbf{P}_{[0, t]}^{\dagger} \mathbf{P}_{[t, 0]}$, is separated from the other eigenvalues then the power iteration sequence $\mathbf{b}_{k}=\mathbf{H} \mathbf{b}_{k-1} /\left\|\mathbf{b}_{k-1}\right\|$, converges to the eigenvector $\dot{\mathbf{u}}_{p}$ corresponding to $\lambda^{*}$ provided that $\left\langle\mathbf{b}_{k}, \mathbf{b}_{0}\right\rangle \neq 0 .\left\langle\mathbf{b}_{k}, \mathbf{H} \mathbf{b}_{k}\right\rangle /\left\langle\mathbf{b}_{k}, \mathbf{b}_{k}\right\rangle$, converges to $\lambda=G^{2}$.

${ }^{11}$ D. S. Henningson, A. Lundbladh, and A. V. Johansson, "A mechanism for bypass transition from localized disturbances in wall-bounded shear flows," J. Fluid Mech. 250, 169 (1993).

${ }^{12}$ M. Chevalier, Ph.D. thesis, Royal Institute of Technology, Department of Mechanics, Stockholm, Sweden, 2004, downloadable at www.mech.kth.se

${ }^{13}$ L. H. Gustavsson, "Energy growth of three-dimensional disturbances in plane Poiseuille flow," J. Fluid Mech. 224, 241 (1991).

${ }^{14}$ P. Andersson, L. Brandt, A. Bottaro, and D. Henningson, "On the breakdown of boundary layers streaks," J. Fluid Mech. 428, 29 (2001).

${ }^{15}$ L. Brandt, C. Cossu, J.-M. Chomaz, P. Huerre, and D. S. Henningson, "On the convectively unstable nature of optimal streaks in boundary layers," J. Fluid Mech. 485, 221 (2003).

${ }^{16} \mathrm{C}$. Cossu and L. Brandt, "On Tollmien-Schlichting waves in streaky boundary layers," Eur. J. Mech. B/Fluids 23, 815 (2004). 